\title{
Robustness and assortativity for diffusion-like processes in scale-free networks
}

\author{
G. D’Agostino ${ }^{1}$, A. Scala ${ }^{2,3(a)}$, V. Zlatić ${ }^{4}$ and G. Caldarelli ${ }^{5,3}$ \\ ${ }^{1}$ ENEA - CR "Casaccia" - Via Anguillarese 301, I-00123, Roma, Italy, EU \\ ${ }^{2}$ CNR-ISC and Department of Physics, University of Rome "Sapienza" - P.le Aldo Moro 5, I-00185, Rome, Italy, EU \\ ${ }^{3}$ London Institute of Mathematical Sciences - 22 South Audley St., Mayfair, London W1K 2NY, UK, EU \\ ${ }^{4}$ Theoretical Physics Division, Rudjer Bošković Institute - P.O. Box 180, HR-10002 Zagreb, Croatia \\ ${ }^{5}$ IMT Lucca Institute for Advanced Studies - Piazza S. Ponziano 6, Lucca, I-55100, Italy, EU
}

received 3 November 2011; accepted in final form 21 February 2012

published online 20 March 2012

PACS 89.75. Hc - Networks and genealogical trees

PACS 05.70.Ln - Nonequilibrium and irreversible thermodynamics

PACS 87.23.Ge - Dynamics of social systems

\begin{abstract}
By analysing the diffusive dynamics of epidemics and of distress in complex networks, we study the effect of the assortativity on the robustness of the networks. We first determine by spectral analysis the thresholds above which epidemics/failures can spread; we then calculate the slowest diffusional times. Our results shows that disassortative networks exhibit a higher epidemiological threshold and are therefore easier to immunize, while in assortative networks there is a longer time for intervention before epidemic/failure spreads. Moreover, we study by computer simulations the sandpile cascade model, a diffusive model of distress propagation (financial contagion). We show that, while assortative networks are more prone to the propagation of epidemic/failures, degree-targeted immunization policies increases their resilience to systemic risk.
\end{abstract}

Copyright (C) EPLA, 2012

Introduction. - The heterogeneity in the distribution of contacts in a population is one of the key factors affecting the propagation of diseases [1]. For example, a large variance of the degree (the number of a node neighbours) distribution is a typical feature of complex networks $[2,3]$ that plays a role in determining the dynamical process defined on the networks itself [4]. It has been shown [5,6] that in the presence of a large heterogeneity, the value of epidemic threshold tends to vanish in the limit of infinitely large network leading therefore to a finite probability of pandemic outbreak $[7,8]$. Such results have been obtained for specific models of diffusion processes as SIR and SIS on complex networks $[1,6,9]$. Similar considerations apply to the analysis of any kind of propagation, as that of financial distress, even if in the latter case the channels of propagation are different from the epidemiological ones. Most of the derivation of the analytical results are based on the mean-field hypothesis and on the analysis of the spectral properties of suitable matrices associated with the network $[6,10-12]$ (we describe that matrices in

(a) E-mail: antonio.scala@phys . uniroma1.it detail in the following). This kind of studies is particular useful in order to define suitable procedures to stop the propagation of an epidemic [13-15].

In comparison to the humongous efforts that have been devoted to understand the role of the distribution of contacts in the networks, less attention has been paid to the assortativity (or vertex-vertex degree correlation) of the networks. Actually, most if not all real networks have non-trivial values of this vertex-vertex correlation. In particular, some networks exhibit "assortative mixing" on their degrees, i.e. high-degree vertices tend to be attached to high-degree vertices; other networks show "disassortative mixing", i.e. high-degree vertices tend to be attached to small-degree vertices. The network's degree-degree correlation can be summarized by a single scalar quantity $r$ called the assortativity coefficient [16]. This quantity assumes the value $r=0$ for degree-uncorrelated networks, is $r>0$ for assortative networks and $r<0$ for disassortative ones. Assortative correlations are typically observed in social networks [16], while disassortative connections are mainly found in technological and biological networks [17]. 
We produce and analyse different networks with the same degree sequence but different assortativity. We first focus on spectral analysis, a very general approach to determine the diffusion dynamics on a complex network by matrix analysis; in particular, we derive the difference in epidemics propagation for networks with different assortativity properties. We then focus on a classical model of distress propagation, the BTW sandpile [18]; using computer simulations, we study the effectiveness of targeted immunization policies with respect to the assortativity of the underlying network.

Methods. - Formally, a network (or a graph) is defined as a pair $G=(V, E)$, where $V$ is the set of $N_{V}$ nodes and $E$ is the set of $N_{E}$ links; each link joins two nodes. To each graph $G$ we associate its adjacency matrix $A$, defined as $A_{i j}=1$ if nodes $i, j$ are connected, $A_{i j}=0$ otherwise. We consider networks that are simple (no self loops, i.e. $\left.A_{i i}=0\right)$ and undirected $\left(A_{i j}=A_{j i}\right)$. The degree of node $i$ is the number of its neighbours and is defined as $k_{i}=\sum_{j} A_{i j}$. The Laplacian matrix of a network is defined as $\mathcal{L} \equiv K-A$, where $K$ is the diagonal matrix of degrees $K_{i j}=k_{i} \delta_{i j}$. The Laplacian matrix is the analogous of the Laplacian operator and describes the diffusion of random walkers on the network.

The assortative coefficient $r$ is the degree-degree Pearson correlation coefficient of two vertices connected by an edge $r=\left[\langle k q\rangle-\langle(1 / 2)(k+q)\rangle^{2}\right] /\left[\left\langle(1 / 2)\left(k^{2}+q^{2}\right)\right\rangle-\right.$ $\left.\langle(1 / 2)(k+q)\rangle^{2}\right]$, where $k, q$ are the degrees of two adjacent vertices; averages $\langle\cdots\rangle$ are over the edges. In order to sample the space of possible networks with respect to the assortativity, we use the procedure of ref. [19].

We sample the statistical ensemble of graphs $\{G\}$ with probability measure $\mu(G) \propto e^{-H(G)}$ induced by the Hamiltonian $H(G)=-J \sum_{i j} A_{i j} k_{i} k_{j}$, where $J$ is the coupling constant measuring the strength of the interaction.

In order to sample configurations according to $\mu(G)$, we explore the configuration space by link rewiring [20] and accept a link rewiring with probability $e^{-\Delta H}$. Once the initial network is given, such procedure leaves the initial degree sequence unchanged. We explore 21 equally spaced values of $J$ ranging from -10 to 10 producing 100 independent configurations for each $J$. Since $H \propto\left\langle k_{i} k_{j}\right\rangle$, the average $H / J$ sampled in this statistical ensemble, decreases if the assortativity increases and vice versa (fig. 1). Notice that the values of the assortativity $r$ with respect to the parameter $J$ is monotonously increasing (fig. 1, inset).

Spectral analysis. - While the sampling procedure is general, in this paper we focus on initial network configurations obtained by the Barabási-Albert preferential attachment (BA networks) [21]. For each value of $J$, we average over $10^{2}$ networks of $10^{4}$ nodes each.

We first calculate the maximum eigenvalue $\Lambda_{1}$ of the adjacency matrices. The adjacency matrix $A$ dictates which nodes can be immediately reached by a "virulent" node and is hence central in describing not only the

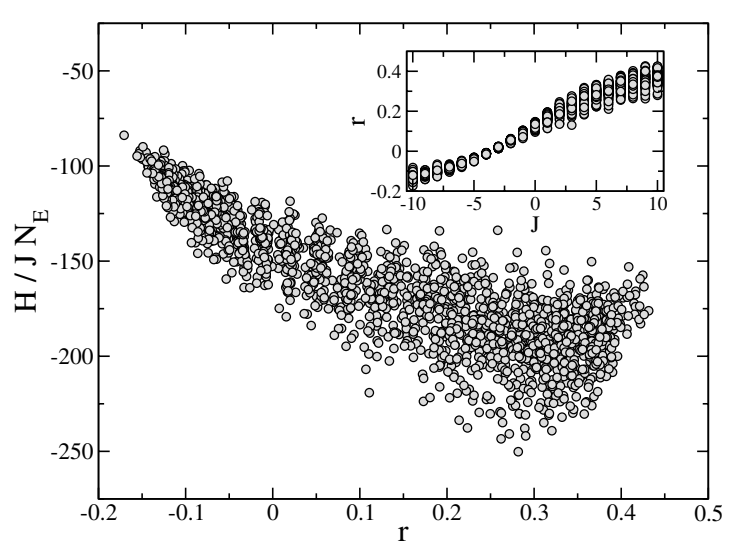

Fig. 1: Values of the energy per link $H / J N_{E}$, vs. the assortativity coefficient (i.e. degree-degree correlation) $r$, for all the 2100 networks of 10000 nodes. In the inset the coefficient $r$ for the same networks as a function of $J$.

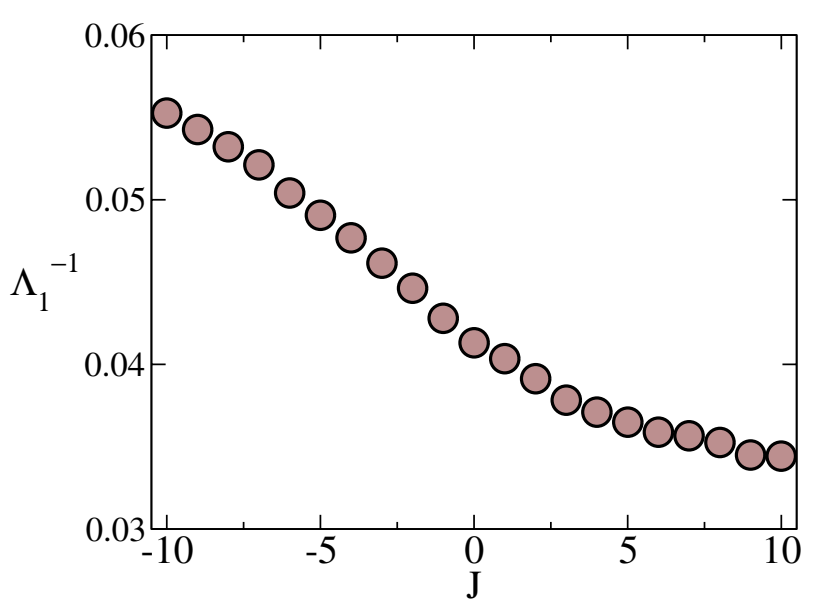

Fig. 2: (Color on-line) $\Lambda_{1}^{-1}$ vs. $J$. The decrease of $\Lambda_{1}^{-1}$ with $J$ and therefore with assortativity indicates a lower epidemic threshold; hence disassortative networks are less prone to epidemic spreading.

propagation of epidemics but also the propagation of faults/failures [11]; its maximum eigenvalue $\Lambda_{1}$ is related to how fast a process can spread in a network.

For the SIS model of infections, Wang and coauthors have shown that the epidemic threshold $\tau$ of a network is exactly $\tau=\Lambda_{1}^{-1}[11] ; \tau$ is defined as the critical ratio among the propagation rate and the recovery rate of a disease above which epidemics ensue.

We find that $\Lambda_{1}^{-1}$ decreases with assortativity: in the range of correlation explored, the most disassortative networks show an epidemic threshold about $60 \%$ higher than most assortative ones (fig. 2). Our findings confirm the idea that avoiding direct connections between hubs (highly connected nodes) may provide protection against epidemics [22].

We then calculate the first non-zero eigenvalue $\lambda_{2}$ of the Laplacian matrices. The Laplacian $\mathcal{L}$ describes the 


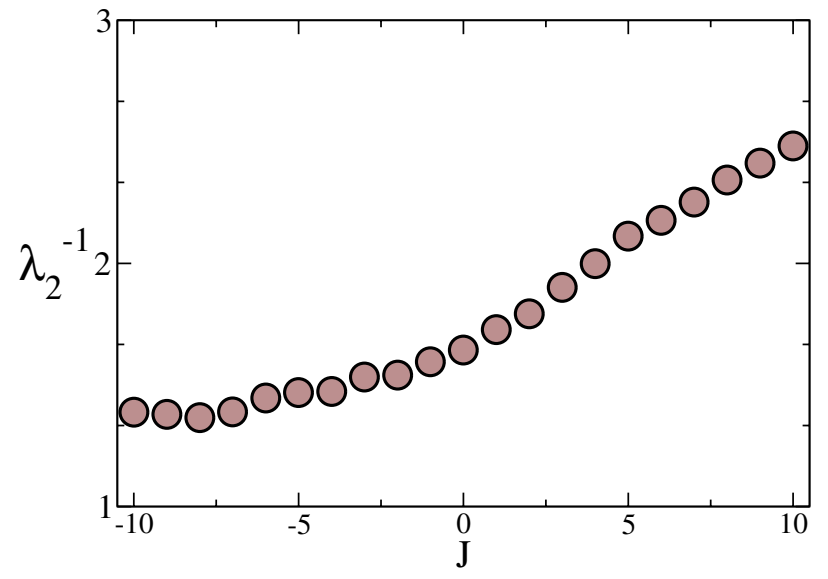

Fig. 3: (Colour on-line) $\lambda_{2}^{-1}$ vs. J. The increase of $\lambda_{2}^{-1}$ with $J$ and therefore with assortativity indicates a growth of the longest relaxation time for diffusion processes on the network. Analogously, both the slowest vibrational mode and the synchronization time increase with assortativity.

diffusive process $\partial_{t} \rho=-\mathcal{L} \rho$ on the network. While the first eigenvalue $\lambda_{1}=0$ is associated to the stationary distribution, the first non-zero eigenvalue $\lambda_{2}$ is the inverse timescale of slowest mode of diffusion.

In general, we can think of $\lambda_{2}^{-1}$ as the longest timescale after which a perturbation (like the infection of a site) that spreads diffusively will settle a new state (like an epidemics) in the network. Therefore, a small value of $\lambda_{2}^{-1}$ means that there is less time for intervention before a network is totally compromised by randomly propagating failures or epidemics; in this respect most assortative networks show times up to $80 \%$ higher than most disassortative ones (fig. 3 ).

The eigenvalues of $\mathcal{L}$ govern also the harmonic dynamics of a network: the adimensional vibration equation in the node displacements $x$ can in fact be written as $\partial_{t}^{2} \mathbf{x}=-\sum_{j} A_{i j}\left(x_{i}-x_{j}\right)=-\mathcal{L} \mathbf{x}$ and again the period of the slowest oscillatory mode is $\lambda_{2}^{-1}$. Almendral and coauthors have shown [23] that in general synchronization times in complex networks have an almost linear dependence on $\lambda_{2}^{-1}$.

Distress propagation. - Beside epidemiological models, there are other types of contagion propagation paradigms that are especially suitable for accounting cascades of financial distress. Avalanche dynamics or domino effect (as it is frequently indicated in economics) is currently regarded as an important feature during financial crises. These phenomena are frequently modelled by means of a class of cellular automata known as sandpile models [18] that can mimic financial distress propagation [24]; financial crisis fluctuations are described, at least qualitatively, by the avalanches of a sandpile model [25]. Conservation laws provide the connection between sandpile models and random-walk (diffusive) models [26].
In sandpiles every vertex has a given capacity to store a scalar field (originally "sand"). For our purposes, such a scalar can represent financial distress (debts) or the probability of a failure. When such a quantity reaches a threshold value, the vertex becomes "bankrupt" and passes its distress to the neighbours. Similarly to previous studies [27-29] we set the failure threshold equal to the degree $k$ of vertex. The simulation time is discrete and at every step we add a grain of sand (distress) on a randomly drawn vertex. When the threshold is reached, the vertex topples and distributes a grain of sand (unit of distress) to every neighbour. Topplings continue as long as some vertex is above threshold; a single toppling can therefore produce an avalanche. The size $s$ of an avalanche is defined as the number of topplings occurring until there are no more vertices above threshold. When the avalanche stops, we add new grains until a new avalanche starts and so on. With respect to the original formulation, here we assume that a fraction $P$ of the vertices are immunized and that such vertices can absorb infinite amount of sand (distress); this is the analogous to say that a vertex is under the coverage of a central bank. To each strategy of choosing the immunized vertices corresponds a policy aimed to limit avalanche propagation, a feature of particularly relevance in the case of a financial crisis; it is anyhow important to use the least number of immunized vertices to minimize the financial cost.

By using this cellular automaton we determine the effects of the assortativity on different policies to stop the propagation of distress; in particular, we consider both a random and a targeted policy of vertex immunization.

In the random policy we pin $P N_{V}$ randomly chosen vertices; for such a case, assortativity does not play a significant role and the fraction $P$ of pinned nodes (i.e. nodes that can absorb any amount of distress) is the only control parameter.

For small $P$ avalanche sizes are power-law distributed with an exponent $\gamma=3 / 2[30]$; this is the worst scenario, where avalanches of the size of the system (systemic crisis) can occur.

The avalanche size frequency takes the functional form $f(s) \propto s^{-3 / 2} e^{-s / \xi}$; the cutoff $\xi$ is a measure of the largest possible avalanches and increases with $P$. From the point of view of the policy maker, it is crucial to limit the size $\xi$ of the systemic crises keeping the minimum effort (i.e. small $P$ ).

In the targeted immunization policy we sort the vertices according to their degrees and pin the first $P N_{V}$ starting from the largest hub. Such policy takes into account the fragility of the hubs in power-law networks [31]. At fixed $P$, we find that targeted immunization is always more efficient than the random one; moreover, the effects of the assortativity become evident even for small values of $P$. In fig. 4 we present the avalanche size distribution for the targeted immunization on networks with large $(J=10)$ and small $(J=-10)$ assortativity. While the exponent of the power law remains $\gamma=3 / 2$ for both 


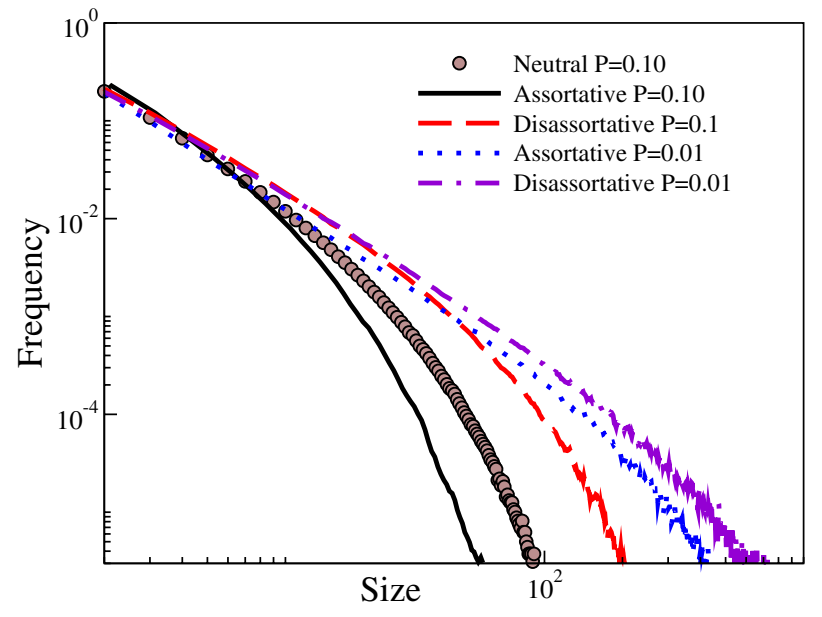

Fig. 4: (Colour on-line) Avalanche size frequency distribution for an ensemble of 100 networks of 10000 nodes each; for each network, we immunise (pin) the $P N_{V}$ nodes with largest degrees. In the figure we compare the most assortative networks with the most disassortative ones. As expected, increasing $P$ mitigates systemic cascades by depressing the probability of the biggest avalanches, i.e. decreasing the cut-off values $\xi$. At fixed $P$, we observe a clear improvement between the cut-off values $\xi$ for assortative networks with respect to disassortative ones, i.e. the size of the largest avalanche is smaller. As an example, immunizing $10 \%(P=0.1)$ of the banks, assortativity improves the effectiveness of the policy by factor $\sim 4$. Such an effect is monotonous: as an example, the avalanche frequencies at $P=0.10$ for neutral $(r \sim 0)$ networks has an intermediate behavior respect to the assortative and the disassortative cases.

immunization policies, we find that the value of the cutoff $\xi$ strongly depends on the assortativity of the network. In particular, assortative networks are subject to much smaller systemic crisis than disassortative ones; therefore, assortative networks are easier to immunise.

Conclusions. - In this paper we considered the effects of the topology on the propagation of diseases or distress in a network system by means of spectral analysis and simulations. This problem is often approached by considering the statistical distribution of the number of contacts, we instead focused on the two-point degree correlation.

First, we show that assortativity increases the probability of a pandemic (low epidemic threshold) while decreasing the speed of diffusive exploration (slow diffusive modes).

Second, we find that in a simple model mimicking financial distress propagation, targeted immunization policy may limit the size of financial crisis; we find that assortativity strongly enhances the effectiveness of such a policy. These results can be used to devise efficient and fast actions to protect infrastructural networks of any kind.

We believe that this paper contributes to a better understanding of immunization procedures on complex networks and to a better evaluation of the robustness of a given system. We find that assortativity cannot be regarded as a topological intrinsic improvement: it enhances time for intervention and improves the effectiveness of financial immunization policies, but epidemics/failures are easier to propagate. Our findings indicate that policy makers in financial markets need to account carefully for the assortativity of the network to mitigate the spread of economic crises in financial markets.

$$
* * *
$$

We thank Prof. L. Braunstein for pointing out ref. [19]. AS, VZ and GC acknowledge support from FET Open Project "FOC" No. 255987. GD acknowledges support by the European project "MOTIA" JLS-2009CIPS-AG-C1-016. AS and GC acknowledge support from the grant HDTRA1-11-1-0048.

\section{REFERENCES}

[1] Pastor-Satorras R. and Vespignani A., Phys. Rev. Lett., 86 (2001) 3200.

[2] Albert R. and Barabási A.-L., Rev. Mod. Phys., 74 (2002) 47.

[3] Caldarelli G., Scale-free Networks (Oxford University Press) 2007.

[4] Buldyrev S. V., Parshani R., Paul G., Stanley H. E. and Havlin S., Nature, 464 (2010) 1025.

[5] Boguñá M., Pastor-Satorras R. and Vespignani A., Phys. Rev. Lett., 90 (2003) .

[6] Durrett R., Proc. Natl. Acad. Sci. U.S.A., 107 (2010) 4491.

[7] Eubank S., Vullikanti A., Khan M., Marathe M. and Barrett C., Beyond degree distributions: Local to global structure of social contact graphs, in Advances in Social Computing, edited by Chai S.-K., Salerno J. and Mabry P., Lect. Notes Comput. Sci., Vol. 6007 (Springer, Berlin, Heidelberg) 2010, p. 1.

[8] Yin Y., Zhang D., Pan G., He M. and Tan J., Phys. Scr., 76 (2007) 606.

[9] Castellano C. and Pastor-Satorras R., Phys. Rev. Lett., 105 (2010) 218701.

[10] Boguná M. and Pastor-Satorras R., Phys. Rev. E, 66 (2002) 047104.

[11] Wang Y., Chakrabarti D., Wang C. and Faloutsos C., Epidemic spreading in real networks: An eigenvalue viewpoint, in Proceedings of the 22nd Symposium on Reliable Distributed Systems, 6-8 October 2003, Florence, Italy (IEEE) 2003, pp. 25-34.

[12] Chakrabarti D., Wang Y., Wang C., Leskovec J. and Faloutsos C., ACM Trans. Inf. Syst. Secur., 10 (2008) 1.

[13] Moreno Y., Gómez J. B. and Pacheco A. F., Phys. Rev. E, 68 (2003) 035103.

[14] Badham J. and Stocker R., Theor. Popul. Biol., 77 (2010) 71.

[15] Kiss I. Z., Green D. M. and Kao R. R., J. R. Soc. Interface, 5 (2008) 791.

[16] Newman M. E. J., Phys. Rev. Lett., 89 (2002) 208701. 
[17] Newman M. E. J., Phys. Rev. E, 67 (2003) 026126.

[18] Bak P., Tang C. and Wiesenfeld K., Phys. Rev. Lett., 59 (1987) 381.

[19] NoH J., Phys. Rev. E, 76 (2007) 026116.

[20] Maslov S. and Sneppen K., Science, 296 (2002) 910.

[21] Barabási A.-L. and Albert R., Science, 286 (1999) 509.

[22] Eguíluz V. M. and Klemm K., Phys. Rev. Lett., 89 (2002) 108701.

[23] Almendral J. A. and Diaz-Guilera A., New J. Phys., 9 (2007) 187.

[24] Feigenbaum J., Rep. Prog. Phys., 66 (2003) 1611.
[25] Bartolozzi M., Leinweber D. and Thomas A., Physica A, 370 (2006) 132.

[26] Shilo Y. and Biham O., Phys. Rev. E, 67 (2003) 066102.

[27] Chen W. and D'Souza R. M., Phys. Rev. Lett., 106 (2011) 115701.

[28] Goh K.-I., Lee D.-S., Kahng B. and Kim D., Phys. Rev. Lett., 91 (2003) 148701.

[29] Lee K.-M., Yang J.-S., Kim G., Lee J., Goh K.-I. and KIM I.-M., PLoS ONE, 6 (2011) e18443.

[30] Alstrøm P., Phys. Rev. A, 38 (1988) 4905.

[31] Albert R., Jeong H. and Barabási A.-L., Nature, 406 (2000) 200. 